\title{
Impact of lockdown on emergency general surgery during first 2020 COVID-19 outbreak
}

\author{
Hayato Kurihara ${ }^{1}$ (D) Enrico Marrano ${ }^{1} \cdot$ Martina Ceolin $^{1} \cdot$ Osvaldo Chiara $^{2} \cdot$ Roberto Faccincani $^{3} \cdot$ Pietro Bisagni $^{4}$.

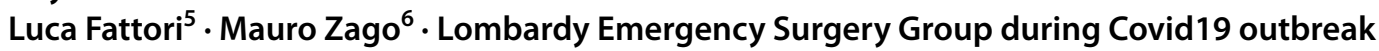

Received: 12 February 2021 / Accepted: 29 April 2021 / Published online: 4 May 2021

○) Springer-Verlag GmbH Germany, part of Springer Nature 2021

\begin{abstract}
Purpose To evaluate and analyze the impact of lockdown strategy due to coronavirus disease 2019 (COVID-19) on emergency general surgery (EGS) in the Milan area at the beginning of pandemic outbreak.

Methods A survey was distributed to 14 different hospitals of the Milan area to analyze the variation of EGS procedures. Each hospital reported the number of EGS procedures in the same time frame comparing 2019 and 2020 . The survey revealed that the number of patients during the COVID-19 pandemic outbreak in 2020 was reduced by $19 \%$ when compared with 2019. The decrease was statistically significant only for abdominal wall surgery. Interestingly, in 2020 , there was an increase of three procedures: surgical intervention for acute mesenteric ischemia $(p=0.002)$, drainage of perianal abscesses $(p=0.000285)$, and cholecystostomy for acute cholecystitis $(p=0.08)$.

Conclusions During the first COVID-19 pandemic wave in the metropolitan area of Milan, the number of patients operated for emergency diseases decreased by around $19 \%$. We believe that this decrease is related either to the fear of the population to ask for emergency department (ED) consultation and to a shift towards a more non-operative management in the surgeons 'decision making' process. The increase of acute mesenteric ischaemia and perianal abscess might be related to the modification of dietary habits and reduction of physical activity related to the lockdown.
\end{abstract}

Keywords COVID-19 outbreak · Emergency general surgery $\cdot$ Emergency department $\cdot$ Lockdown effect

\section{Introduction}

The members of Lombardy Emergency Surgery Group during Covid19 outbreak are listed in aknowledgements.

Hayato Kurihara

hayato.kurihara@humanitas.it

1 Emergency Surgery and Trauma Section, Department of Surgery, IRCCS, Humanitas Research Hospital, Rozzano, Italy

2 Universita' di Milano, Chirurgia Generale-Trauma Team ASST Niguarda, Milano, Italy

3 Pronto Soccorso e Chirurgia Generale e Delle Urgenze, IRCCS Ospedale San Raffaele, Milano, Italy

4 Ospedale Maggiore di Lodi-ASST Lodi, Lodi, Italy

5 Dipartimento di Chirurgia-Chirurgia d'Urgenza, Ospedale San Gerardo ed Universita' Degli Studi Milano-Bicocca, Monza, Italy

6 General and Emergency Surgery Division, Robotic and Emergency Surgery Department, A. Manzoni Hospital, ASST Lecco, Lecco, Italy
Due to the outbreak of coronavirus disease 2019 (COVID19) in Northern Italy at the end of February 2020, a containment strategy was immediately established by Regional authorities to limit the spread of the virus. To increase medical surge capacity to treat patients with novel severe acute respiratory syndrome coronavirus 2 (SARS-CoV2) and reduce the in-hospital spreading of the virus, the population was invited not to report spontaneously to the Emergency Department (ED) unless for severe symptoms. The first Italian patient tested as positive for novel coronavirus was reported in Codogno Hospital (Lodi, Lombardy, Italy) on February 20, 2020. After an initial local restriction of a "red zone" in the epicentral area of the virus outbreak, from March 9, 2020, the lockdown was extended at regional and national level. The lockdown termination was originally planned for April 3, 2020, but was then extended to May 3, 2020 [1]. In an effort to optimize surge capacity, especially regarding intensive-care units' beds, the Regional Crisis Unit 
of Lombardy remodeled the emergency system establishing hub centers dedicated to trauma, stroke, cardiovascular diseases, and single skeletal trauma. Furthermore, some hospitals were dedicated to surgical oncology for patients with clinical priorities, while all scheduled surgery for benign diseases was temporarily cancelled [2].

The containment policy led to a general reduction of access to the ED, both emergency general surgery (EGS) and trauma cases. A similar scenario, in terms of reduction of ED access, has already been reported in the UK where, after the announcement of a lockdown, the numbers of accident and emergency attendances consistently dropped down [3]. Although a reduction of admittance to ED of injury-related cases can be easily explained as a direct consequence of mobility limitations as well as industrial discontinuation, many surgeons of Northern Italy reported a reduction of non-traumatic acute surgical conditions where diagnosis and treatment delay play a crucial role on optimal surgical outcome (acute appendicitis, bowel obstruction, acute biliary disease, incarcerated hernia, or hollow viscus perforation). These concerns and personal perspectives have been shared among different surgical units in the Milan and Lombardy area through web-based meetings. There was a consensus on the need to investigate this potentially dangerous trend. The surgeons involved in the discussion agreed to compare the flow of EGS cases that presented to ED in a time frame during the outbreak of COVID-19 and the same time frame of the previous year, when COVID-19 did not impact yet on the Italian population. A survey was therefore prepared by the Emergency Surgery and Trauma Unit of IRCCS, Humanitas Research Hospital, and then distributed to 14 different hospitals of the Milan area, to assess the actual decrease of cases with EGS-related conditions and analyze the differences for specific acute surgical conditions.

\section{Materials and methods}

A survey was prepared by the Emergency Surgery and Trauma Unit of IRCCS Humanitas Research Hospital and distributed to 14 hospitals of an area surrounding Milan and Central-West Lombardy (Fig. 1), including major and peripheral institutes (Table 1). The survey regarded the time period from 21st of February through 3rd of April. All hospitals included in the survey had a General Surgery Unit and an Emergency Department with open access to selfreporting patients. All hospitals were active during COVID19 pandemic both as COVID and Non-COVID centers. Data
Fig. 1 Geographical distribution of hospitals involved in the survey

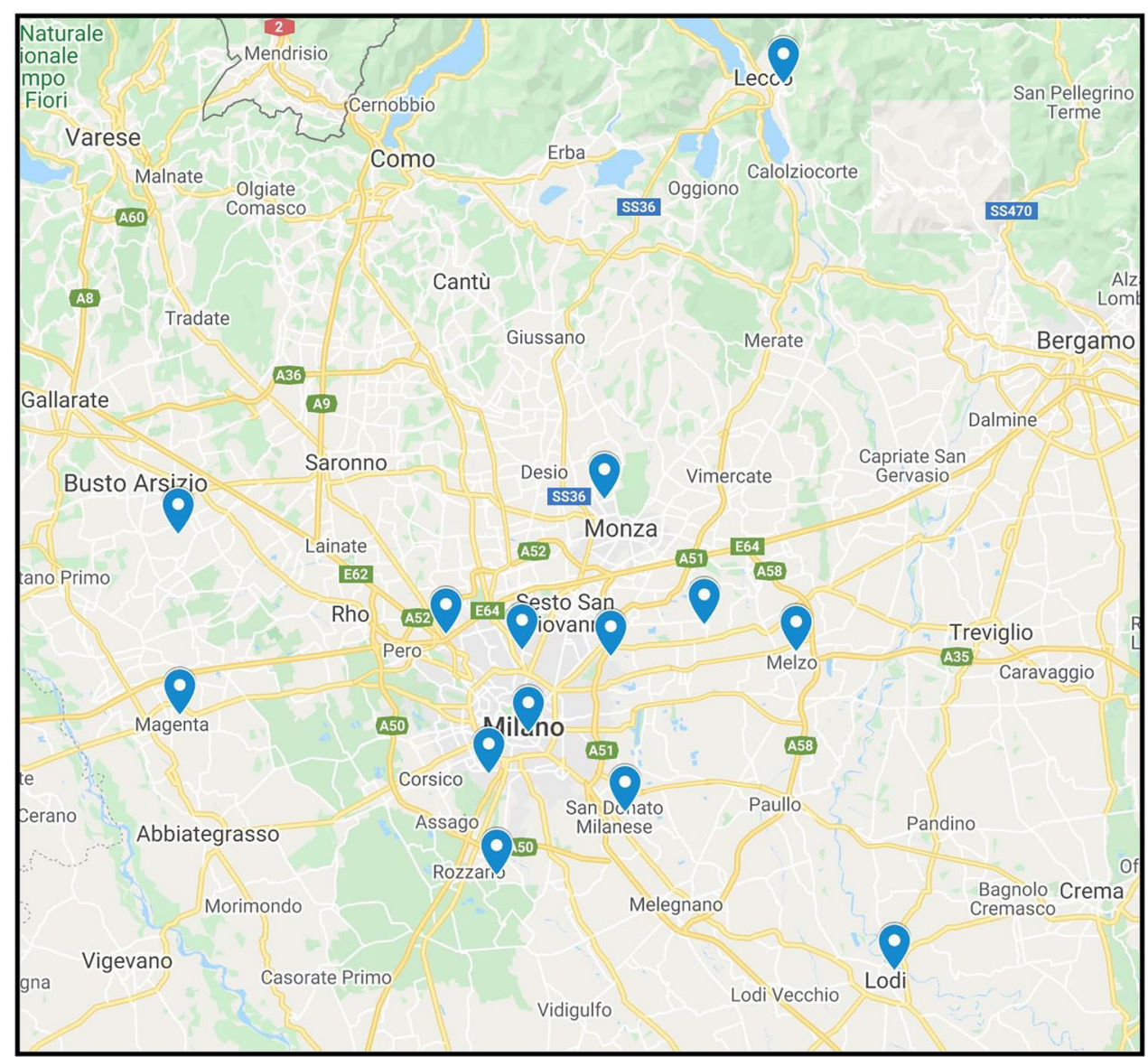


Table 1 Hospitals joining the survey

\begin{tabular}{lll}
\hline Hospital name & Location & Province \\
\hline IRCCS, Humanitas Research Hospital & Rozzano & Milan \\
ASST Fatebenefratelli & Milano & Milan \\
Ospedale A. Manzoni & Lecco & Lecco \\
Ospedale di Legnano & Legnano & Milan \\
Ospedale Maggiore di Lodi & Lodi & Lodi \\
Ospedale G. Fornaroli & Magenta & Milan \\
Ospedale Santa Maria delle Stelle & Melzo & Milan \\
ASST Grande Ospedale Niguarda & Milano & Milan \\
Ospedale Maggiore Policlinico & Milano & Milan \\
Ospedale Sacco & Milano & Milan \\
IRCCS Policlinico San Donato & San & Milan \\
& Donato & \\
Ospedale San Gerardo & Milanese & \\
Ospedale San Paolo & Monza & Monza e Brianza \\
IRCCS Ospedale San Raffaele & Milano & Milan \\
\hline
\end{tabular}

collections focused on comparing the amount of emergency surgical procedures performed in a selected period in 2020 versus the same time frame in 2019.

Each hospital was asked to report the number of patients who underwent emergency general surgery procedures during the same time frame (February 21-April 3) in 2020 and 2019, respectively.

Analyzed surgical procedures:

- Acute appendicitis,

- Acute cholecystitis,

- Small bowel and large bowel obstruction,

- Bowel perforation,

- Acute complicated diverticulitis,

- Upper gastrointestinal perforation or hemorrhage,
- Acute mesenteric ischaemia,

- Incarcerated or strangulated groin or incisional hernia,

- Anorectal abscess.

Surgical interventions for trauma patients were not included.

Inclusion criteria were: patients $\geq 18$ years, surgery performed within $72 \mathrm{~h}$ from ED access, patients transferred from other hospitals.

Exclusion criteria were: in-hospital emergencies, procedures for postoperative complications. Non-operative management was excluded with the exception of percutaneous and endoscopic cholecystostomy.

COVID-19 infection status of patients undergoing emergency operations was reported as negative, suspected, or positive based on the result of nasal/pharyngeal swab, pharyngeal aspirate or broncho-alveolar lavage, and chest CT scan or lung ultrasound.

Our endpoint was to confirm our suspicion of reduction in the number of patients requiring EGS procedures.

The survey was distributed through a Microsoft Office Excel file, while Chi-square test was used for the qualitative data analysis; $p$ value $\leq 0.05$ was considered statistically significant.

\section{Results}

All 14 hospitals answered our survey and data from 844 patients were included. The number of patients of the control group in 2019 was higher $(n=468)$ than the number of patients observed in the same time frame during the COVID19 pandemic outbreak in $2020(n=376)$. The corresponding reduction was 92 patients $(-19 \%)$, as shown in Table 2 .

Before surgical intervention, each patient was screened for SARS-CoV-2 nucleic acid detection. The combination of
Table 2 Emergency general surgery procedures in the same time frame (2019 vs. 2020 pandemic outbreak)

\begin{tabular}{lrrrr}
\hline Surgical procedures & 2019 & 2020 & Delta $\%$ & $p$ \\
\hline Appendectomy & 118 & 81 & -31.36 & 0.21 \\
Cholecystectomy & 88 & 61 & -30.68 & 0.32 \\
Cholecystostomy and endoscopic cholecystostomy & 2 & 6 & 200.00 & 0.08 \\
Surgery for small bowel obstruction & 58 & 43 & -25.86 & 0.66 \\
Surgery for large bowel obstruction & 25 & 22 & -12.00 & 0.74 \\
Surgery for bowel perforation (not diverticular disease) & 27 & 23 & -14.81 & 0.83 \\
Surgery for complicated acute diverticulitis & 22 & 18 & -18.18 & 0.95 \\
Surgery for gastroduodenal bleeding or perforation & 15 & 13 & -13.33 & 0.83 \\
Surgery for acute mesenteric ischemia & 12 & 26 & 116.67 & 0.002000 \\
Surgery for incarcerated groin hernia & 45 & 22 & -51.11 & 0.044000 \\
Surgery for strangulated incisional or umbilical hernia & 27 & 10 & -62.96 & 0.028000 \\
Anorectal abscess drainage & 29 & 51 & 75.86 & 0.000285 \\
Total & 468 & 376 & -19.66 & \\
\hline
\end{tabular}


the results of radiology and laboratory discriminated patients as COVID positive, negative, or suspected. At the beginning of the outbreak, due to the high volume of patients, the results of the microbiology test required a long time and seven patients, suspected of COVID-19 infection, could not wait for the swab result due to critical condition. These patients, all with a thoracic CT scan with ground glass pattern, underwent surgery in a dedicated COVID operating theater and followed a COVID-positive pathway,

Among the 376 patients operated between February 21 st and April 3rd 2020, 3 patients (0.8\%) were COVID positive and 7 patients $(1.86 \%)$ were COVID-19 suspected at the time of surgery.

Although the number of patients operated in the 2019 was generally higher than in 2020, the reduction of cases was statistically significant only for incarcerated groin hernia and incisional/umbilical hernia. The number of incarcerated groin hernia was 22 in the 2020 and 45 in $2019(p=0.044)$, likewise the number of incarcerated incisional or umbilical hernia ( $n=10$ vs. $27, p=0.028$ ).

On the other hand, three emergency general surgery procedures increased their frequencies: surgical intervention for acute mesenteric ischemia (26 in 2020 vs 12 in $2019,+117 \%, p=0.002$ ), drainage of perianal abscesses (51 in 2020 vs 29 in $2019,+76 \%, p=0.000285$ ), and cholecystostomy for acute cholecystitis (6 in 2020 vs 2 in $2019,+200 \%, p=0.08)$.

\section{Discussion}

Our survey from 14 different hospitals in the Milan area confirmed an overall decrease of the most common emergency general surgery procedures during the COVID-19 pandemic 2020 period compared to the same time frame in 2019 ( $n=376$ vs. $n=468,-19 \%$ ).

Although statistically not significant, this result is similar to the one reported by Gallego et al., who described a decrease in the number of emergency general surgery interventions from an average of 3.6 to 1.6 per day [4]. Recently, a report from Rausei et al. showed similar results in the Lombardy area [5].

Although we do not have a clear reason to explain the overall decrease of EGS cases, particularly in case of lifethreatening situations, we assume that the population feared being infected from COVID-19 and were reluctant to ask for medical consultation to the ED. The second factor that might explain the decrease of emergent surgical procedure is the shift of surgeons' decision-making towards a more conservative non-operative management to exceed resource capacity or to avoid patients to risk of COVID-19 infection.

The analysis of subgroups of emergency surgical interventions showed a statistically significant decrease of incarcerated abdominal wall hernia cases; in particular, for groin hernias the difference was $n=22$ vs. $n=45(p=0.044)$, while in case of incarcerated incisional and umbilical hernias was $n=10$ vs. $n=27$ ( $p=0.028)$. A possible explanation might be the lockdown that forced the population to stay at home, with reduction of physical activity and de-escalation of symptoms related to hernia condition. Another possible explanation is the attempt by the population to treat at home the incarcerated hernia with an independent conservative management. A third explanation may be related to the attempt of conservative management in the surgical decision-making pathway, but unfortunately, our survey did not collect the number of ED accesses, and therefore, it was not possible to estimate the ratio of non-operative cases related to the patients' admission volume.

Although statistically not significant, we observed an interesting reduction of the number of surgical procedures for multiple emergency surgical conditions as appendectomy for acute appendicitis, surgery for bowel obstructions, and acute diverticulitis (Table 2). We could not collect the data on the overall admissions in the EDs, and therefore, we cannot prove that a conservative management was clearly chosen by surgeons, even though highly suspected by the authors. Moreover, we assume a decrease of patients' admission, as confirmed by direct experience and from the study of Rausei [5].

Although among the surgeons involved in this study, there was a general agreement on not changing the surgical strategy in acute abdominal conditions, as recommended by the European Society for Trauma and Emergency Surgery [6] during the pandemic, we observed an increase use of percutaneous cholecystostomy ( $n=6$ vs $n=2,+200 \%$ ) and a decrease of cholecystectomy ( $n=61$ vs $=88,-30.7 \%$ ), for acute cholecystitis. Although both results are not statistically significant, these trends probably confirm our suspicion of a shift toward conservative treatment rather than a surgical approach for acute cholecystitis and other acute surgical conditions. As we reported above, we did not analyze the number of patients who presented to the ED and have been treated with simple conservative treatment representing one of the major limitations of our study.

Two clinical conditions followed by emergency surgical procedures showed a statistically significant increase: acute mesenteric ischemia ( $n 26$ vs $n 12,+117 \%, p=0.002$ ) and anorectal abscesses ( $n 51$ vs $n 29,+76 \%, p=0.000285$ ). We do not have a clear explanation for the increase of cases with acute mesenteric ischemia. The first hypothesis is the thrombogenic effect of COVID-19 inflammation, well documented by Ignat et al. [7, 8]. They described a higher incidence of small bowel ischemia associated with coronavirus infection, but in our population, most of the operated patients were COVID-19 negative and is therefore not possible to assume a relation with the infection. Another possible explanation 
could be the change of dietary habit during the lockdown; an increased food uptake was in fact informally reported by the population during the National lockdown. Di Rienzo et al. confirmed it with the analysis of a survey distributed by institutional and private social networks in Italy, where the perception of weight gain was observed in $48.6 \%$ of the survey respondents [9]. The hypothesis is that an increase of food intake associated with a decrease in physical aerobic activities during the lockdown could have impacted on the bowel blood demand and consequent development of mesenteric ischemia. However, up to now, it remains unclear why there was a reduction in these life-threatening situations where urgent surgery is mandatory.

Surprisingly, our survey revealed a statistically significant increase of surgical procedures also for anorectal abscesses. In this case, we suppose that there could be a relation with an increase of food intake and sedentary habits, forced by the containment strategy and lockdown.

\section{Study limitations}

We are aware of the limitations related to the retrospective nature of the study and the lack of detailed data regarding overall volume access to EDs with subgroup information for each disease. With this bias, it is of course not possible to correctly explain the reasons for the decrease of EGS procedures, but the survey emphasized the impact of the COVID19 outbreak on our health system on surgical emergencies.

\section{Conclusions}

The decrease of EGS procedures might be explained by the fear of the population to get access to the ED; furthermore, we suppose a shift versus a non-operative approach in surgeons' decision-making process that might explain the higher number, although statistically not significant, of higher cholecystostomy for acute cholecystitis. Reduction in surgical interventions for abdominal wall hernias might be influenced either by a less aggressive surgical strategy, but also by less physical activity. Nevertheless, it remains unclear why there was a reduction in life-threatening situations where urgent surgery is mandatory. Conversely, the statistically significant increase of surgical interventions for acute mesenteric ischemia and anorectal abscesses might be explained by the forced "stay-at-home" policy with consequent less physical activity and variation of dietary habits.

Acknowledgements HK would like to thank Dr. Matteo Porta for being a flexible rod. Lombardy Emergency Surgery Group during COVID-19 outbreak: Andrea Spota (Università di Milano, Chirurgia GeneraleTrauma Team ASST Niguarda, Milano); Michele Carlucci, Federico Pascucci (Pronto Soccorso e Chirurgia Generale e delle Urgenze,
IRCCS Ospedale San Raffaele, Milano); Francesca Teodora Armao, Marco Longhi (Ospedale Maggiore di Lodi, ASST Lodi); Maria Masutti (Dipartimento di Chirurgia-Chirurgia d'Urgenza, Ospedale San Gerardo ed Università degli Studi Milano-Bicocca, Monza); Marco Mariani e Ludovica Gibelli (General \& Emergency Surgery Division, Robotic \& Emergency Surgery Department, A. Manzoni Hospital, ASST Lecco); Diego Mariani (Ospedale Nuovo Legnano, ASST Ovest Milanese, Legnano); Laura Castoldi (Department of Emergency Surgery, Foundation IRCCS Ca' Granda Ospedale Maggiore Policlinico, Milano); Piergiorgio Danelli, Claudio Guerci, Anna Maffioli (Scuola di Specializzazione in Chirurgia Generale, Dipartimento di Scienze biomediche e cliniche Università degli Studi di Milano, U.O.C Chirurgia Generale, Dipartimento di area Chirurgica, ASST Fatebenefratelli Sacco, Chirurgia Generale Sacco, Milano); Antonella Ardito, Giulio Iacob (Chirurgia Fatebenefratelli, ASST Fatebenefratelli Sacco, Milano); Matteo Marconi, Giuliano Sarro (General and Emergency Surgery, Ospedale G. Fornaroli, ASST Ovest Milanese, Magenta); Andrea Casamassima (Chirurgia Generale, Ospedale S. Maria delle Stelle, ASST Melegnano e della Martesana PO Melzo, Melzo); Saverio Chiaravalle (Servizio di Pronto Soccorso, IRCCS Policlinico San Donato, Milano); Nicolò Mariani (ASST Santi Paolo e Carlo, Milano).

Funding The authors received no funding for this research.

\section{Declarations}

Conflict of interest The authors declare no competing financial interest.

Research involving human participants and/or animals This article does not contain any studies with human participants or animals performed by any of the authors.

Informed consent For this type of study, formal consent was not required.

\section{References}

1. Grasselli G, Pesenti A, Cecconi M. Critical care utilization for the COVID-19 outbreak in Lombardy, Italy: early experience and forecast during an emergency response. JAMA. 2020;323(16):1545-6. https://doi.org/10.1001/jama.2020.4031.

2. Kurihara H, Bisagni P, Faccincani R, Zago M. COVID-19 outbreak in Northern Italy: view-point of the Milan area surgical community. J Trauma Acute Care Surg. 2020;88(6):719-24. https://doi.org/10.1097/TA.0000000000002695.

3. Thornton J. Covid-19: A\&E visits in England fall by $25 \%$ in week after lockdown. BMJ. 2020;369:m1401. https://doi.org/10.1136/ bmj.m14014.

4. Álvarez Gallego M, de Gortázar Las Casas S, Pascual Migueláñez I, Rubio-Pérez I, Barragán Serrano C, Álvarez Peña E, Díaz Domínguez J. SARS-CoV-2 pandemic on the activity and professionals of a general surgery and digestive surgery service in a tertiary hospital. Cir Esp. 2020;98(6):320-7 (Epub 2020 Apr 7).

5. Rausei S, Ferrara F, Zurleni T, Frattini F, Chiara O, Pietrabissa A, Sarro G; for Italian Associa- tion of Hospital Surgeons (ACOI). Dramatic decrease of surgical emergencies during COVID-19 outbreak. J Trauma Acute Care Surg. 2020. https://doi.org/10.1097/ TA.0000000000002923 (Epub ahead of print).

6. Coimbra R, Edwards S, Kurihara H, et al. European society of trauma and emergency surgery (ESTES) recommendations for trauma and emergency surgery preparation during times of 
COVID-19 infection. Eur J Trauma Emerg Surg. 2020. https:// doi.org/10.1007/s00068-020-01364-7.

7. Connors JM, Levy JH. COVID-19 and its implications for thrombosis and anticoagulation. Blood. 2020;135(23):2033-40.

8. Ignat M, Philouze G, Aussenac-Belle L, Faucher V, Collange $\mathrm{O}$, Mutter D, et al. Small bowel ischemia and SARS-CoV-2 infection: an underdiagnosed distinct clinical entity. Surgery. 2020;168(1):14-6.
9. Di Renzo L, Gualtieri P, Pivari F, et al. Eating habits and lifestyle changes during COVID-19 lockdown: an Italian survey. J Transl Med. 2020;18(1):229. https://doi.org/10.1186/ s12967-020-02399-5. 